\title{
CERTAIN GAMES, CATEGORY, AND MEASURE
}

\author{
SLAWOMIR SOLECKI
}

(Communicated by Andreas R. Blass)

\begin{abstract}
For $A \subset 2^{\omega}$ and $X \subset \omega$ consider an infinite game $\Gamma(A, X)$ in which two players I and II choose $c_{n} \in\{0,1\} . c_{n}$ is chosen by I if $n \in X$ and by II if $n \in \omega \backslash X$. I wins if $\left(c_{0}, c_{1}, c_{2}, \ldots\right) \in A$. We analyze connections between $A$ and the family of all sets $X \subset \omega$ for which $I$ has a winning strategy in $\Gamma(A, X)$. Certain similarities and differences appear if one formulates these connections in the language of category and of Lebesgue measure.
\end{abstract}

\section{Notation}

The set of all natural numbers is denoted by $\omega$. Each natural number is the set of its predecessors. For any set $X$ by $2^{X}$ we denote the set of all functions from $X$ to $2=\{0,1\}$ and by $\mathscr{P}(X)$ the set of all subsets of $X$. There is a canonical correspondence between the elements of $\mathscr{P}(X)$ and $2^{X}$ : a subset of $X$ corresponds to its characteristic function. If $f \in 2^{X}$ and $Y \subset X$, then $f \mid Y$ denotes the restriction of $f$ to $Y$. For $F \subset 2^{X}$ (resp., $F \subset \mathscr{P}(X)$ ) and $Y \subset X$ let $F \mid Y=\{f \mid Y: f \in F\} \quad$ (resp., $F \mid Y=\{A \cap Y: A \in F\}$ ). If $f \in 2^{n}$ then $[f]=\left\{g \in 2^{\omega}: g \mid n=f\right\}$, and if $F \subset 2^{n}$ then $[F]=\bigcup_{f \in F}[f]$. By $X \triangle Y$ we denote the symmetric difference of $X$ and $Y$ and by $|X|$ the cardinality of $X$.

Mostly we will deal with $2^{\alpha}$ and $\mathscr{P}(\alpha)$ for $\alpha \leq \omega .2^{\omega}$ is naturally equipped with the product measure of measures on 2 assigning weight $\frac{1}{2}$ to both points from 2. This product measure is denoted by $\lambda$ and is called Lebesgue measure. By the same letter we denote the measure on $\mathscr{P}(\omega)$ obtained by pulling back $\lambda$ from $2^{\omega}$. $\lambda_{*}$ stands for the inner measure. We also have a natural metric on $2^{\alpha}, \alpha \leq \omega$, defined by the formula $d\left(f_{1}, f_{2}\right)=2^{-n}$ where $n=$ $\sup \left\{k<\alpha: f_{1}\left|k=f_{2}\right| k\right\}$ for $f_{1} \neq f_{2}$. Again, by the same letter we denote the metric on $\mathscr{P}(\alpha)$ obtained by pulling back $d$. The metric $d$ on $2^{\alpha}$ (resp., $\mathscr{P}(\alpha))$ induces a metric on $\mathscr{K}\left(2^{\alpha}\right)$ (resp., $\mathscr{K}(\mathscr{P}(\alpha))$ ), the family of all compact subsets of $2^{\alpha}$ (resp., $\left.\mathscr{P}(\alpha)\right)$, by Hausdorff's formula

$$
d\left(F_{1}, F_{2}\right)=\max \left\{\sup _{f \in F_{1}} d\left(f, F_{2}\right), \sup _{f \in F_{2}} d\left(f, F_{1}\right)\right\}
$$

where $d(f, F)=\inf \{d(f, g): g \in F\}$.

Received by the editors January 27, 1992.

1991 Mathematics Subject Classification. Primary 04A99; Secondary 90D05.

Key words and phrases. Infinite games, category, measure. 


\section{INTRODUCTION}

Given $A \subset 2^{\omega}$ and $X \subset \omega$ let us consider the following infinite game with perfect information $\Gamma(A, X)$ (cf. [1,3]). Players I and II choose $c_{n} \in 2=$ $\{0,1\}, n \in \omega$. If $n \in X$ then $c_{n}$ is chosen by I and if $n \in \omega \backslash X$ by II. We say that $\mathrm{I}$ wins if $\left(c_{0}, c_{1}, c_{2}, \ldots\right) \in A$.

Define $\Gamma_{A}=\{X \in \mathscr{P}(\omega)$ : I has a winning strategy in $\Gamma(A, X)\}$. By a winning strategy for I we mean a function $\varphi: 2^{<\omega} \mapsto 2$ such that if $c_{n}, n \in \omega$, are chosen so that $c_{n}=\varphi\left(c_{0}, \ldots, c_{n-1}\right)$ for $n \in X$ (if $0 \in X, c_{0}=\varphi(\varnothing)$ ), then $\left(c_{0}, c_{1}, c_{2}, \ldots\right) \in A$, i.e., no matter how II plays, if I plays according to $\varphi$, then I wins. A winning strategy for II can be defined similarly.

Obviously the bigger $A$ is the more games $\Gamma(A, X), X \subset \omega$, are won by I, i.e., the bigger $\Gamma_{A}$ is. We will try to make this statement more precise. In [1, Theorem 1.4] Balcerzak and Roslanowski implicitly proved the following theorem. (Their Theorem 1.4 states more or less the same as Corollary 2.2 here.)

Theorem 2.1. If $A \subset 2^{\omega}$ contains a set of second category possessing the Baire property then there exist $k \in \omega$ and a partition $\left\{X_{n}: n \in \omega\right\}$ of $\omega \backslash k$ into finite sets such that $\left\{k \cup \bigcup_{n \in Y} X_{n}: Y \subset \omega\right.$ infinite $\} \subset \Gamma_{A}$. If $A$ is residual, we can put $k=0$.

From this the following two corollaries can easily be drawn.

Corollary 2.1. If $A$ contains a set of second category with the Baire property, so does $\Gamma_{A}$. If $A$ is residual, then $\Gamma_{A}$ is residual, too.

Corollary 2.2. If $A$ is residual, then $\Gamma_{A}$ contains an infinite family of disjoint subsets.

The above results estimate the size of $\Gamma_{A}$ provided the size of $A$ in terms of category is known. In this paper we investigate the size of $\Gamma_{A}$ assuming that we know how big $A$ is in terms of Lebesgue measure. In particular, we prove that the result analogous to Corollary 2.1 holds. On the other hand, by an example we show that the result analogous to Corollary 2.2 is false. This answers a question of Balcerzak and Rosłanowski posed in [1, Problem 1.5(a)]. Additionally we prove that if $F$ is closed so is $\Gamma_{F}$ and the mapping $F \mapsto \Gamma_{F}$ considered as a mapping from $\mathscr{K}\left(2^{\omega}\right)$ to $\mathscr{K}(\mathscr{P}(\omega))$ does not increase the distance.

\section{SOME COMBINATORICS}

Let $A \subset 2^{\alpha}$ where $\alpha \leq \omega$. We define a set $\hat{A} \subset \mathscr{P}(\alpha)$ (the operation $:$ is denoted by the same symbol for various $\alpha$ ) by the formula

$$
\begin{aligned}
X \in \widehat{A} \text { iff } \exists F^{\prime} \subset A\left(F^{\prime} \mid X=2^{X} \& \forall f_{1}, f_{2} \in F^{\prime}\right. \\
\left.\left(f_{1} \neq f_{2} \Rightarrow \sup \left\{k \in \alpha: f_{1}\left|k=f_{2}\right| k\right\} \in X\right)\right) .
\end{aligned}
$$

We say that $F^{\prime}$ witnesses $X \in \widehat{A}$. Notice that such an $F^{\prime}$ is closed. Sometimes we write $A$ rinstead of $\widehat{A}$. 
Lemma 3.1. Let $F_{1}, F_{2} \subset 2^{n}, n \in \omega$. Then $d\left(\widehat{F}_{1}, \widehat{F}_{2}\right) \leq d\left(F_{1}, F_{2}\right)$.

Proof. By symmetry it is enough to show that for any $X \in \widehat{F}_{1} \backslash \widehat{F}_{2}$ there is $f \in F_{1} \backslash F_{2}$ with $d\left(X, \widehat{F}_{2}\right) \leq d\left(f, F_{2}\right)$. If $d\left(X, \widehat{F}_{2}\right)=2^{-k}$, then $\min X \triangle Y \leq k$ for any $Y \in \widehat{F}_{2}$. Let $F^{\prime} \subset F_{1}$ witness $X \in \widehat{F}_{1}$. There is $f \in F^{\prime}$ such that $f|(k+1) \neq g|(k+1)$ for all $g \in F_{2}$. If not, there would exist $Y \in \widehat{F}_{2}$ with $Y=X \cap(k+1)$ whence $\min X \triangle Y>k$, a contradiction. For this $f$ we have $d\left(f, F_{2}\right) \geq 2^{-k}$.

The next lemma seems to be interesting in its own right.

Lemma 3.2. Let $F \subset 2^{n}, n \in \omega$. Then $|\widehat{F}|=|F|$.

Proof. Put $\left(f_{1}, f_{2}\right)=\max \left\{k \in n: f_{1}\left|k=f_{2}\right| k\right\}$.

Claim. For any $F \subset 2^{n}$, if $f \in 2^{n} \backslash F$ then $\left.\mid(F \cup\{f\})\right\} \geq|\widehat{F}|+1$.

Assume we have proved the claim. An obvious corollary of it is that if $F, F_{1} \subset 2^{n}$ and $F \cap F_{1}=\varnothing$ then $\left.\mid\left(F \cup F_{1}\right)\right\rceil \geq|\widehat{F}|+\left|F_{1}\right|$. We also have $\widehat{2^{n}}=2^{n}$ and $\hat{\varnothing}=\varnothing$. Therefore

$$
\left.\left|\widehat{2^{n}}\right|=\mid\left(F \cup\left(2^{n} \backslash F\right)\right)\right\rceil \geq|\widehat{F}|+\left|2^{n}\right|-|F|
$$

whence $|\widehat{F}| \leq|F|$. And on the other hand

$$
|\widehat{F}|=|(\varnothing \cup F) \uparrow \geq| \hat{\varnothing}|+| F|=| F \mid \text {. }
$$

Proof of the claim. Let $F_{k}=\{g \in F: g|k=f| k\} \cup\{f\}$. Let $X \subset n$ be such that for each $k \in n, X \backslash k$ is maximal with respect to the inclusion in $\widehat{F}_{k}$. Such a set exists because for any $Y$ maximal in $\widehat{F}_{k+1}$ there exists $Z \supset Y$ maximal in $\widehat{F}_{k}(Z=Y$ or $Z=Y \cup\{k\})$. Therefore $X$ can be constructed inductively with respect to $k$ starting from $n$ and ending at 0 .

We show that $X \in(F \cup\{f\})^{\curlyvee} \widehat{F}$. Obviously, $X \in(F \cup\{f\})^{\wedge}$ as $F \cup\{f\}=F_{0}$ and $X=X \backslash 0 \in \widehat{F}_{0}$. Assume $X \in \widehat{F}$ and let $F^{\prime} \subset F$ witness this. Define $p=\max \left\{(f, g): g \in F^{\prime}\right\}$.

Case 1. $p \in X$. There exist $g_{1}, g_{2} \in F^{\prime}$ with $p=\left(g_{1}, g_{2}\right)$ and $g_{1} \mid p=$ $g_{2}|p=f| p$; but then $f\left|(p+1)=g_{i}\right|(p+1)$ for some $i \in\{1,2\}$, whence $\left(f, g_{i}\right) \geq p+1$, which contradicts the definition of $p$.

Case 2. $p \notin X$. We will apply the following fact: if $Y \in \widehat{F^{1}}$ and $Y \in \widehat{F^{2}}$ and there is $k \in n$ such that $k=\left(g_{1}, g_{2}\right)$ for any $g_{1} \in F^{1}$ and $g_{2} \in F^{2}$, then $Y \cup\{k\} \in\left(F^{1} \cup F^{2}\right)^{\wedge}$. Let $F^{1}=F^{\prime} \cap F_{p}$. Then $X \backslash p \in \widehat{F^{1}}$. Let $F^{2} \subset F_{p+1}$ witness $X \backslash p=X \backslash(p+1) \in \widehat{F_{p+1}}$. Notice that $f|p=g| p$ for any $g \in F^{1} \cup F^{2}$, $f|(p+1) \neq g|(p+1)$ for $g \in F^{1}$, and $f|(p+1)=g|(p+1)$ for $g \in F^{2}$, i.e., $p=\left(g_{1}, g_{2}\right)$ for any $g_{1} \in F^{1}, g_{2} \in F^{2}$. Therefore $(X \backslash p) \cup\{p\} \in\left(F^{1} \cup F^{2}\right)^{\wedge} \subset$ $\widehat{F}_{p}$, which contradicts the maximality of $X \backslash p$ in $\widehat{F}_{p}$.

We summarize this section in the following proposition.

Proposition 3.1. If $F \subset 2^{\omega}$ is closed then $\widehat{F}$ is closed, too. The mapping $F \mapsto \widehat{F}$ from $\mathscr{K}\left(2^{\omega}\right)$ to $\mathscr{K}(\mathscr{P}(\omega))$ does not increase the distance, i.e., $d\left(\widehat{F}_{1}, \widehat{F}_{2}\right) \leq$ $d\left(F_{1}, F_{2}\right)$ for $F_{1}, F_{2} \in \mathscr{K}\left(2^{\omega}\right)$. Moreover, $\lambda(\widehat{F})=\lambda(F)$ for $F \in \mathscr{K}\left(2^{\omega}\right)$.

Proof. For $f_{1}, f_{2} \in 2^{\omega}$ put $\left(f_{1}, f_{2}\right)=\sup \left\{k \in \omega: f_{1}\left|k=f_{2}\right| k\right\}$. Let

$$
\begin{aligned}
\mathscr{R}=\left\{H \in \mathscr{K}\left(2^{\omega}\right): \forall n \in \omega\right. & \left(\exists f_{1}, f_{2} \in H\left(f_{1}, f_{2}\right)=n\right. \\
& \left.\left.\Rightarrow \forall f \in H \exists f^{\prime} \in H\left(f, f^{\prime}\right)=n\right)\right\} .
\end{aligned}
$$


Clearly $\mathscr{R} \subset \mathscr{K}\left(2^{\omega}\right)$ is closed. Let $\phi: \mathscr{K}\left(2^{\omega}\right) \mapsto \mathscr{P}(\omega)$ be defined by $\phi(H)=$ $\left\{n \in \omega: \exists f_{1}, f_{2} \in H\left(f_{1}, f_{2}\right)=n\right\}$. Clearly $\phi$ is continuous. It is easy to see that $\widehat{F}=\phi\left(\mathscr{R} \cap\left\{H \in \mathscr{K}\left(2^{\omega}\right): H \subset F\right\}\right)$. (If $F^{\prime} \subset F$ witnesses $X \in \widehat{F}$ then $F^{\prime}$ is closed; moreover, $F^{\prime} \in \mathscr{R}$ and $X=\phi\left(F^{\prime}\right)$.) Since $\mathscr{R} \cap\left\{H \in \mathscr{K}\left(2^{\omega}\right): H \subset F\right\}$ is compact and $\phi$ is continuous, $\widehat{F}$ is compact.

Claim. Let $F \subset 2^{\omega}$ be closed. Then $\bigcap_{n=1}^{\infty}[\widehat{F \mid n}]=\widehat{F}$.

Proof of the claim. Let $X \in \widehat{F} \mid n$, i.e., there is $X_{1} \in \widehat{F}$ with $X_{1} \cap n=X$. But then if $F^{\prime} \subset F$ witnesses $X_{1} \in \widehat{F}$ then $F^{\prime} \mid n$ witnesses $X \in \widehat{F \mid n}$. So $\widehat{F} \mid n \subset \widehat{F \mid n}$ and $\bigcap_{n=1}^{\infty}[\widehat{F \mid n}] \subset \bigcap_{n=1}^{\infty}[\widehat{F} \mid n]=\widehat{F}$.

Now, let $X \in \bigcap_{n=1}^{\infty}[\widehat{F \mid n}]$, i.e., $X \cap n \in \widehat{F \mid n}$ for each $n \in \omega$. The set $T=\left\{F^{\prime}: \exists n \in \omega F^{\prime} \subset F \mid n\right.$ and $F^{\prime}$ witnesses $\left.X \cap n \in \widehat{F \mid n}\right\}$ with the relation $F^{\prime}<F^{\prime \prime}$ iff $F^{\prime}=F^{\prime \prime} \mid n$ for some $n \in \omega$, for $F^{\prime}, F^{\prime \prime} \in T$, constitutes an infinite tree each level of which is finite. By König's lemma there exists an infinite branch, i.e., a sequence $F_{n}^{\prime} \in T, n \in \omega$, with $F_{n}^{\prime}<F_{n+1}^{\prime}$. Then $F^{\prime}=\bigcap_{n=1}^{\infty}\left[F_{n}^{\prime}\right] \subset F$ and $F^{\prime}$ witnesses $X \in \widehat{F}$. Thus the claim is proved.

Now assume that $F_{1}, F_{2}$ are closed. Since $\widehat{F}_{1}, \widehat{F}_{2}$ are closed, we get

$$
d\left(\widehat{F}_{1}, \widehat{F}_{2}\right)=\lim _{n} d\left(\widehat{F_{1} \mid n}, \widehat{F_{2} \mid n}\right) \leq \lim _{n} d\left(F_{1}\left|n, F_{2}\right| n\right)=d\left(F_{1}, F_{2}\right)
$$

by the claim and Lemma 3.1. If $F$ is closed, we get

$$
\lambda(\widehat{F})=\lim _{n}|\widehat{F \mid n}| / 2^{n}=\lim _{n}|F| n \mid / 2^{n}=\lambda(F)
$$

by the claim and Lemma 3.2 .

$$
\text { 4. } \Gamma_{A}
$$

Lemma 4.1. Let $A \subset 2^{\omega}$. Then $X \in \hat{A}$ if and only if $\omega \backslash X \in \Gamma_{A}$.

Proof. $(\Rightarrow)$ Let $F^{\prime}$ witness $X \in \hat{A}$. We have to find a winning strategy $\varphi$ for I in the game $\Gamma(A, \omega \backslash X)$. Let $\sigma \in 2^{n}$ for some $n \in \omega$. If $[\sigma] \cap F^{\prime}=\varnothing$ or $n \in X$, let $\varphi(\sigma)$ be anything, e.g., 0 . If $[\sigma] \cap F^{\prime} \neq \varnothing$ and $n \in \omega \backslash X$, let $f \in F^{\prime}$ be such that $f \in[\sigma] \cap F^{\prime}$. Put $\varphi(\sigma)=f(n)$. The choice of $F^{\prime}$ ensures that $\mathrm{I}$ uses only the second rule in a play. Therefore we have $\left[\left(c_{0}, \ldots, c_{n}\right)\right] \cap F^{\prime} \neq \varnothing$ for any $n \in \omega$. Since $F^{\prime}$ is closed, $\left(c_{0}, c_{1}, c_{2}, \ldots\right) \in F^{\prime} \subset A$.

$(\Leftarrow)$ Let $\varphi$ be a winning strategy for $\mathrm{I}$ in $\Gamma(A, \omega \backslash X)$. Let $F^{\prime}=\{f \in$ $\left.2^{\omega}: \forall n \in \omega \backslash X f(n)=\varphi(f \mid n)\right\}$. It is easy to check that $F^{\prime} \subset A$ and $F^{\prime}$ witnesses $X \in \hat{A}$.

Theorem 4.1. Let $F \subset 2^{\omega}$ be closed. Then $\Gamma_{F}$ is closed and the mapping $F \mapsto \Gamma_{F}$ from $\mathscr{K}\left(2^{\omega}\right)$ to $\mathscr{K}(\mathscr{P}(\omega))$ does not increase the distance.

Proof. Since $\pi: \mathscr{P}(\omega) \mapsto \mathscr{P}(\omega)$ defined by $\pi(X)=\omega \backslash X$ is a distance preserving homeomorphism, we obtain the conclusion by Proposition 3.1 and Lemma 4.1.

Point (ii) in the next theorem is a measure counterpart of Corollary 2.1. A weaker result was proved by Rosłanowski in [4, Lemma 5.6]. It was shown there that if $F$ is closed and $\lambda(F)>0$ then $\Gamma_{F} \neq \varnothing$. 
Theorem 4.2. (i) If $A \subset 2^{\omega}$ is the intersection of an open set and a closed set, then $\Gamma_{A}$ is $F_{\sigma}$, thus measurable, and $\lambda\left(\Gamma_{A}\right)=\lambda(A)$.

(ii) For any $A \subset 2^{\omega}$ we have $\lambda_{*}\left(\Gamma_{A}\right) \geq \lambda_{*}(A)$.

Proof. If $A$ is as in (i) then we can find an increasing sequence of compact sets $F_{n} \subset A$ so that for any compact $F \subset A$ there is $n \in \omega$ with $F \subset F_{n}$. Notice that for each $X \in \Gamma_{A}$ there is a compact set $F \subset A$ with $X \in \Gamma_{F}$. (Take simply $F$ witnessing $\omega \backslash X \in \widehat{A}$.) Thus $\Gamma_{A}=\bigcup_{n=0}^{\infty} \Gamma_{F_{n}}$ whence $\Gamma_{A}$ is $F_{\sigma}$ by Theorem 4.1. Notice also that the sequence $\Gamma_{F_{n}}$ is increasing. Since the mapping $\pi$ defined in the proof of Theorem 4.1 preserves Lebesgue measure, we get

$$
\lambda\left(\Gamma_{A}\right)=\lim _{n} \lambda\left(\Gamma_{F_{n}}\right)=\lim _{n} \lambda\left(\widehat{F_{n}}\right)=\lim _{n} \lambda\left(F_{n}\right)=\lambda(A)
$$

by Lemma 4.1 and Proposition 3.1.

If $A$ is arbitrary, pick a sequence of closed sets $F_{n} \subset A, n \in \omega$, so that $\lim _{n} \lambda\left(F_{n}\right)=\lambda_{*}(A)$. Then $\Gamma_{F_{n}} \subset \Gamma_{A}$ and (ii) follows from (i).

We now draw a corollary which shows that a very weak form of Corollary 2.2 holds for Lebesgue measure, too.

Corollary 4.1. Let $A \subset 2^{\omega}$ be of full measure. Then $\Gamma_{A}$ contains two disjoint subsets.

Proof. By Theorem 4.2 we have $\lambda\left(\Gamma_{A}\right)=1$. Since the mapping $\pi$ defined in the proof of Theorem 4.1 is measure preserving, there is $X \in \pi\left(\Gamma_{A}\right) \cap \Gamma_{A}$, i.e., $X \in \Gamma_{A}$ and $\omega \backslash X \in \Gamma_{A}$.

It is obvious from the proof that the above corollary holds for $A \subset 2^{\omega}$ with $\lambda_{*}(A)>\frac{1}{2}$.

Now we show that in general we cannot find three pairwise disjoint sets in $\Gamma_{A}$ even if $\lambda(A)=1$. This proves that Corollary 2.2 is not true for Lebesgue measure and answers Balcerzak and Rosłanowski's question from [1].

Example. Let

$$
A=\left\{f \in 2^{\omega}: \lim _{n} \frac{|\{k<n: f(k)=1\}|}{n}=\frac{1}{2}\right\} .
$$

By Borel's normal number theorem (see [2, Theorem 1.2 or Theorem 6.1]) $\lambda(A)=1$. Take three pairwise disjoint sets $X_{1}, X_{2}, X_{3} \subset \omega$. There is $i \in\{1,2,3\}$ with $\liminf \inf _{n}\left|X_{i} \cap n\right| / n \leq 1 / 3$. Then $X_{i} \notin \Gamma_{A}$. Indeed, II playing always 0 in $\Gamma\left(A, X_{i}\right)$ forces $\liminf _{n}\left|\left\{k<n: c_{k}=1\right\}\right| / n \leq 1 / 3$ where $\left(c_{0}, c_{1}, c_{2}, \ldots\right)$ is an output of a play. Thus $\left(c_{0}, c_{1}, c_{2}, \ldots\right) \notin A$.

\section{REFERENCES}

1. M. Balcerzak and A. Rosłanowski, On Mycielski ideals, Proc. Amer. Math. Soc. 110 (1990), 243-250.

2. P. Billingsley, Probability and measure, 2nd ed., Wiley, New York, 1986.

3. J. Mycielski, Some new ideals of sets on the real line, Colloq. Math. 20 (1969), 71-76.

4. A. Roslanowski, On games ideals, Colloq. Math. 59 (1990), 159-168.

Mathematical Institute, University of Wroclaw, Pl. Grunwaldzki 2/4, 50-384 WROCLAW, POLAND

Current address: Department of Mathematics, Caltech, Pasadena, California 91125 